\title{
College Health Surveillance Network: Use of Health Services by USA College Students
}

\author{
James C. Turner ${ }^{1}$, Adrienne Keller*1, Jennifer C. Bauerle ${ }^{1}$ and Craig Roberts ${ }^{2}$ \\ ${ }^{1}$ University of Virginia, Charlottesville, VA, USA; ${ }^{2}$ University of Wisconsin-Madison, Madison, WI, USA
}

\section{Objective}

We received CDC funding to create and maintain a multi-institutional de-identified medical records database from student health centers (SHCs) for a nationally representative sample of colleges and universities.

\section{Introduction}

Currently over 18 million students are enrolled in USA institutions of higher education (IHEs), representing more than one-third of the young adult population. In a national survey, about $16 \%$ of students reported living at home [1]. SHCs are therefore an important resource for the majority of college students. College communities are unique settings that are geographically diverse, highly mobile, and densely populated with congregate living and learning conditions. IHEs, therefore, are highly vulnerable to the introduction of contagious diseases with subsequent transmission to surrounding communities. Thousands of counseling and student health centers exist, funded by billions of dollars. Despite these facts, there was no national database on the health care utilization of this population. In an era in which health policies and plans are typically guided by data, we were relatively blind to information about the diagnoses, epidemiologic trends and health care needs of young adults attending colleges and universities.

\section{Methods}

The primary objective was accomplished through five specific, interrelated processes:

1. Expansion of a five school pilot project to approximately 20 IHEs that use electronic medical records (EMRs), with representation in each of the 10 Federal geographic regions;

2. Creation of policies, protocols and procedures to ensure adequate protection of individual identity and medical records information;

3. Creation of specific categories of diagnostic (ICD-9) and service (CPT) codes to capture critical health utilization indicators;

4. Development of inter-institutional agreement on policies and process for use of the data;

5. Establishment and maintenance of a web site that includes a secure area for transmission of data, generation and use of summary data by participating IHEs and feedback from the research team, and a non-secure public access site.

\section{Results}

The College Health Surveillance Network (CHSN) is an online resource of de-identified EMRs for over 702,000 students at 22 USA universities (approximately 900,000 visits per year). This sample of students is highly representative of the 108 four year research institutions nationally. Beginning January 1, 2011, each participating IHE uploads the following data monthly to the secure website: confidential person ID, age, gender, student status, ethnicity/race, date of encounter, ICD-9 codes and CPT codes associated with the encounter. This data is usable in three venues. 1) Participating IHEs can access the website to generate comparative descriptive data. The website allows specification of date range, gender, age range, ethnicity/race, and any one of 109 groups of ICD9 codes or 17 groups of CPT codes. Schools can compare numbers and rates to regional and national data. 2) A public portal allows similar comparisons at the regional and national level. 3) The data is downloaded into a statistical database accessible by the research team for computation of inferential statistics. The presentation will include data on the most prevalent diagnostic categories and types of visits, by demographic characteristics of the students. These data highlight the prominence of mental health concerns among college students seeking medical attention.

\section{Conclusions}

This project provides the first national database specific to the epidemiologic trends and health services utilization for college students and establishes the necessary foundation for identifying the health concerns and preventive health needs of these students as a unique population.

\section{Keywords}

electronic medical records; college students; surveillance

\section{References}

[1] American College Health Association. National College Health Assessment II: Reference Group Executive Summary. Fall 2012. Hanover, MD: American College Health Association 2013.

\footnotetext{
*Adrienne Keller

E-mail: aek3a@virginia.edu
} 